\title{
Barriers and enablers of health system adoption of kangaroo mother care: a systematic review of caregiver perspectives
}

Emily R. Smith", Ilana Bergelson², Stacie Constantian', Bina Valsangkar ${ }^{3}$ and Grace J. Chan ${ }^{1,2}$

\begin{abstract}
Background: Despite improvements in child survival in the past four decades, an estimated 6.3 million children under the age of five die each year, and more than $40 \%$ of these deaths occur in the neonatal period. Interventions to reduce neonatal mortality are needed. Kangaroo mother care (KMC) is one such life-saving intervention; however it has not yet been fully integrated into health systems around the world. Utilizing a conceptual framework for integration of targeted health interventions into health systems, we hypothesize that caregivers play a critical role in the adoption, diffusion, and assimilation of KMC. The objective of this research was to identify barriers and enablers of implementation and scale up of KMC from caregivers' perspective.

Methods: We searched Pubmed, Embase, Web of Science, Scopus, and WHO regional databases using search terms 'kangaroo mother care' or 'kangaroo care' or 'skin to skin care'. Studies published between January 1, 1960 and August 19, 2015 were included. To be eligible, published work had to be based on primary data collection regarding barriers or enablers of KMC implementation from the family perspective. Abstracted data were linked to the conceptual framework using a deductive approach, and themes were identified within each of the five framework areas using Nvivo software.

Results: We identified a total of 2875 abstracts. After removing duplicates and ineligible studies, 98 were included in the analysis. The majority of publications were published within the past 5 years, had a sample size less than 50 , and recruited participants from health facilities. Approximately one-third of the studies were conducted in the Americas, and $26.5 \%$ were conducted in Africa.

We identified four themes surrounding the interaction between families and the KMC intervention: buy in and bonding (i.e. benefits of KMC to mothers and infants and perceptions of bonding between mother and infant), social support (i.e. assistance from other people to perform KMC), sufficient time to perform KMC, and medical concerns about mother or newborn health. Furthermore, we identified barriers and enablers of KMC adoption by caregivers within the context of the health system regarding financing and service delivery. Embedded within the broad social context, barriers to KMC adoption by caregivers included adherence to traditional newborn practices, stigma surrounding having a preterm infant, and gender roles regarding childcare.
\end{abstract}

Conclusion: Efforts to scale up and integrate KMC into health systems must reduce barriers in order to promote the uptake of the intervention by caregivers.

Keywords: Kangaroo mother care, Skin to skin care, Health system integration, Mother, Father, Family, Caregiver

\footnotetext{
*Correspondence: ers231@mail.harvard.edu

'Department of Global Health and Population, Harvard T. H. Chan School of

Public Health, 665 Huntington Ave., Building 1, Boston, MA 02115, USA

Full list of author information is available at the end of the article
} 


\section{Background}

Despite improvements in child survival in the past four decades, an estimated 6.3 million children under the age of five die each year, and more than $40 \%$ of these deaths occur in the neonatal period [1]. Complications related to preterm birth is the leading cause of death among children under five [2]. Effective implementation, at scale, of evidence-based interventions to reduce complications of preterm birth and associated neonatal mortality is needed.

Kangaroo Mother Care (KMC) is one such evidencebased, life-saving intervention. There are four components of KMC including: 1) early, continuous, and prolonged skin-to-skin contact between infant and caregiver, 2) exclusive breastfeeding, 3) early discharge from hospital, and 4) adequate support for caregiver and infant at home $[3,4]$. In addition to providing thermal control, $\mathrm{KMC}$ is associated with a $36 \%$ reduced risk of neonatal mortality among low birth weight newborns compared to conventional care, as well as a significantly reduced risk of sepsis, hypoglycemia, and hypothermia [5].

Despite the strong evidence regarding the improved health outcomes among preterm or low birth weight infants receiving $\mathrm{KMC}$, including a recent recommendation by the World Health Organization that KMC should be routine care for newborns weighing less than $2000 \mathrm{~g}$ [6], this intervention has never been fully integrated into health systems around the world. A previous systematic review identified barriers to health system adoption of KMC and noted that families play an important role in KMC adoption [7]. Further, the review noted that family interactions with the health system were critical to KMC adoption. Caregivers (e.g. mothers, fathers, and families) are key implementers and beneficiaries of KMC. We explore the barriers and enablers of KMC implementation from the caregiver perspective in greater detail.

In order to understand the role of families in the adoption, diffusion, and assimilation of $\mathrm{KMC}$, we build on a conceptual framework for integration of targeted health interventions into health systems [7, 8]. This framework promotes analysis in five areas including: (1) definition of the problem; (2) definition and attributes, such as the 'relative advantage' and 'complexity', of the intervention package; (3) the adoption system including key actors, their interest, values and the power dynamics between them; (4) health system characteristics; and (5) the broad context including demographic, economic, and cultural factors.

Using this framework, we analyzed how caregivers perceive the risks and benefits of the intervention, as well as their values and interests surrounding KMC. Specifically, we identified barriers and enablers of implementation and scale up of kangaroo mother care based on the first systematic review on KMC implementation and uptake from the caregiver perspective.

\section{Methods}

In order to identify research studies for this review, we searched Pubmed, Embase, Web of Science, Scopus, AIM, LILACS, IMEMR, IMSEAR, and WPRIM. Search terms included: 'kangaroo mother care', or 'kangaroo care', or 'skin to skin care'. Studies published between January 1, 1960 and August 19, 2015 were included. We also reviewed the references of published systematic reviews, searched unpublished programmatic reports, and requested data from the Saving Newborn Lives Program at Save the Children. We excluded studies if they did not include human subjects and primary data collection. To be eligible for inclusion into the review, published work had to include information about barriers to or enablers of successful implementation of KMC from the family perspective based on the experience of caregivers and health providers who had implemented KMC.

Two independent reviewers used a standardized data abstraction form to assess eligibility and abstract data from each article. If the case reviewers did not agree about the inclusion of a study, a third reviewer broke the tie. Each eligible study was assessed for the potential risk of bias in five domains including: selection bias, appropriateness of data collection, appropriateness of data analysis, generalizability, and consideration of ethics [9].

Through several iterations of manual annotation and indexing, two researchers coded themes, perspectives and experiences using NVivo software. Abstracted data were linked to the conceptual framework using a deductive approach, and themes were identified within each of the five framework areas. Narratives were constructed around each major theme, and we used quotes to summarize perspectives from each study. The major themes and narratives were used to develop matrices where we defined important concepts, the range and nature of each theme, and the relationship between themes. We used this meta-synthesis approach based on our objective of understanding caregiver perception and our hope that the results can inform policy and enhance our understanding of how to implement this complex intervention within health systems [10].

\section{Results}

We identified a total of 2875 abstracts (748 in Embase, 645 in Scopus, 556 in Pubmed, 518 in Web of Science, 379 in WHO Regional Databases, and 29 from other sources). There were 1360 abstracts after removing duplicates. 716 were excluded after title and abstract review, and the full text was reviewed for 644. A total of 98 were included in the analysis (Fig. 1). All studies were considered of sufficient quality to include in the analysis. 


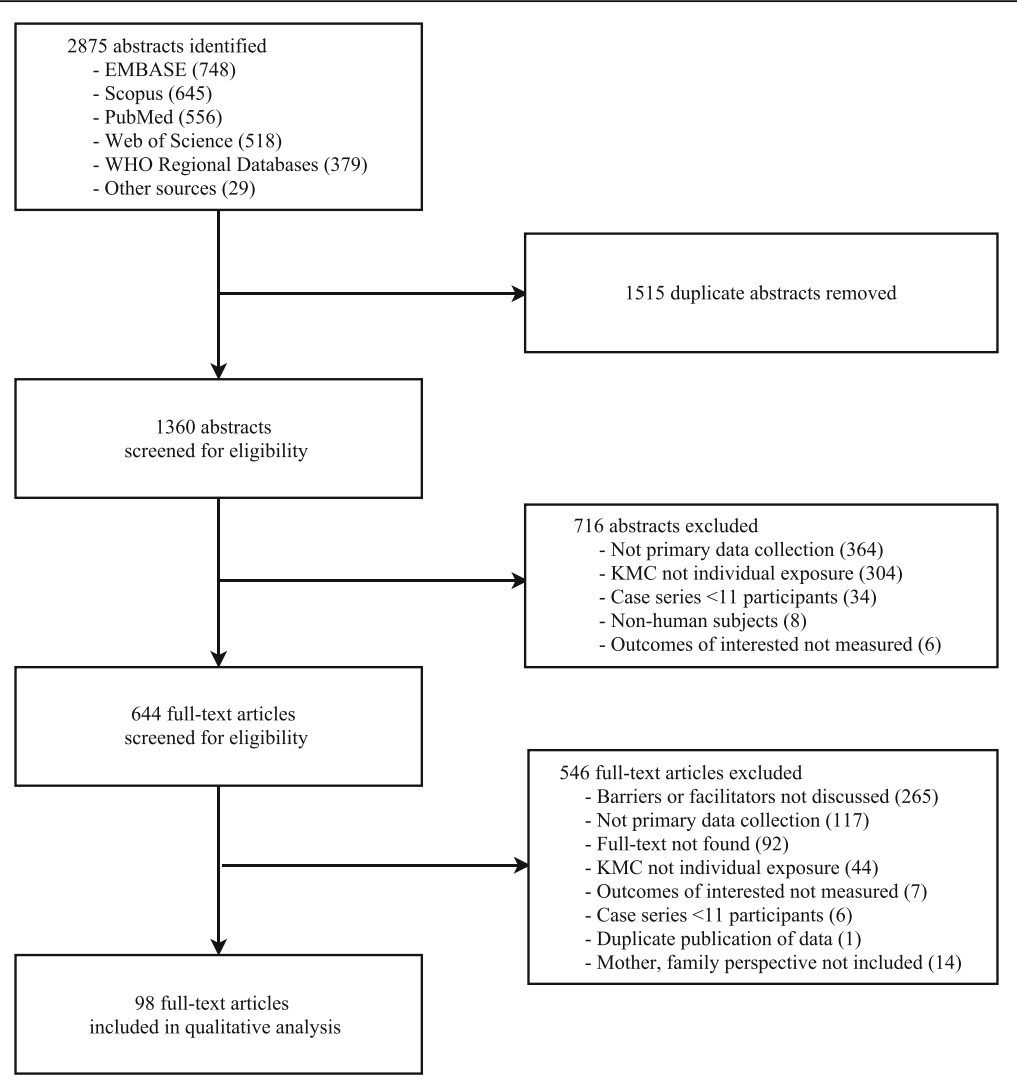

Fig. 1 Systematic review flow chart

The majority of publications were published within the past 5 years, had a sample size less than 50 , and recruited participants from health facilities. One-third of the studies were conducted in the Americas, $26.5 \%$ in Africa, 16.3\% in Europe, and the remaining in Southeast Asia, Eastern Mediterranean, Western Pacific, or in multiple regions. More than half of the studies were conducted in an area with a neonatal mortality rate $<15$ deaths per 1000 live births (Table 1).

After analyzing the data collected from the family perspective, we confirmed that caregivers are an essential component of the KMC adoption system as they are the primary decision-makers and are responsible for infant feeding and skin-to-skin contact. We identified four themes surrounding the interaction between caregivers and the KMC intervention: buy in and bonding, social support, time, and medical concerns. Furthermore, we identified barriers and enablers of KMC adoption by families within the context of the health system and the broader social context. These themes are summarized in Table 2.

\section{Barriers and enablers for caregiver adoption of KMC Caregiver buy-in and bonding}

Buy-in and bonding referred to the acceptance of KMC, belief in the benefits of KMC to mothers and preterm infants, and reported perceptions of bonding between mother and infant.

Uptake of KMC was impaired by limited buy-in to KMC by mothers, fathers, and families. For example: "My experience told me this KMC was not right...so before caesarean section (in the meeting with a neonatal nurse). I was worried about it." (Father) [11]. Mothers were less likely to accept KMC if healthcare workers could not clearly explain the benefits of KMC. Parents reported that they were simply told to perform KMC without explanation why or how to do so, and the feeling that $\mathrm{KMC}$ was forced upon them hindered buy-in from caregivers [12]. Another barrier to parental buy-in occurred when caregivers perceived that their newborn did not enjoy KMC. In some areas due to the hot climate, parents observed their infant became irritable or "stinky" during SSC [13]. Less frequently, caregivers mentioned discomfort at not being able to see their newborn during KMC [14]. Another barrier was lack of bonding by mothers with their preterm infants [11]. In some cases lack of bonding with the infant was due to fear, stigma, shame, guilt, or anxiety about having a preterm infant $[15,16]$, and some did not want to keep the baby at all [17]. For example: "I wished that I had had a miscarriage instead of delivering this preterm, it would 
Table 1 Characteristics of included studies $(N=98)$

\begin{tabular}{|c|c|}
\hline Study Charactersitics & Percent \\
\hline \multicolumn{2}{|l|}{ Year } \\
\hline 2010 to 2014 & 55.1 \\
\hline 2000 to 2009 & 34.7 \\
\hline 1988 to 1999 & 3.1 \\
\hline Missing & 6.1 \\
\hline \multicolumn{2}{|l|}{ Sample Size } \\
\hline$<50$ & 53.1 \\
\hline 50 to $<1000$ & 7.1 \\
\hline 100 to $<200$ & 7.1 \\
\hline$\geq 200$ & 32.7 \\
\hline \multicolumn{2}{|l|}{ NMR (deaths per 1000 live birth) } \\
\hline$<5$ & 31.6 \\
\hline 5 to $<15$ & 24.5 \\
\hline 15 to $<30$ & 32.7 \\
\hline$\geq 30$ & 4.1 \\
\hline Missing & 7.1 \\
\hline \multicolumn{2}{|l|}{ Setting (rural or urban) } \\
\hline Urban & 44.9 \\
\hline Urban and rural & 10.2 \\
\hline Rural & 6.1 \\
\hline Missing & 38.8 \\
\hline \multicolumn{2}{|l|}{ Population source } \\
\hline Health facility & 60.2 \\
\hline NICU or stepdown unit & 27.6 \\
\hline Community or population-based surveillance & 11.2 \\
\hline Missing & 1.0 \\
\hline \multicolumn{2}{|l|}{ Gestational Age } \\
\hline Preterm 34 to $<37$ weeks & 11.2 \\
\hline All gestational ages & 11.2 \\
\hline Very preterm $<34$ weeks & 7.1 \\
\hline Mixed preterm and very preterm $<37$ weeks & 6.1 \\
\hline Full term $\geq 37$ weeks & 4.1 \\
\hline Missing & 60.2 \\
\hline \multicolumn{2}{|l|}{ Birth weight } \\
\hline Low birth weight 1500 to $<2500 \mathrm{~g}$ & 13.3 \\
\hline All birth weights & 12.2 \\
\hline Mixed low and very low birth weight $<2500 \mathrm{~g}$ & 7.1 \\
\hline Very low birth weight $<1500 \mathrm{~g}$ & 4.1 \\
\hline Missing & 63.3 \\
\hline
\end{tabular}

be better. I never thought that this baby would survive; I thought that it would die any time" (Mother) [17].

However, positive perceptions among mothers, fathers, and families regarding the potential benefits of the intervention promoted KMC uptake. Caregivers who successfully implemented KMC perceived that performing KMC calmed their baby [18-22]. These mothers observed their newborns sleeping longer during skin-to-skin contact; infants were described as less anxious, more restful, more willing to breastfeed, and happier to be in SSC position than in an incubator [18, 23]. KMC was also perceived as a healing mechanism for the parents. It helped mothers and fathers recover emotionally and physically, as well as create a family bond [24]. KMC made mothers feel useful. For example: "Every time I hold her, the monitorseverything-did better. Her oxygen SATs did better, I really think I helped her... and think that the human contact and...hearing my heart and everything, I really think that helped her" [19]. Some fathers reported feeling needed and enjoyed participating in the early care of their newborn. Further, families using KMC described the time during and after $\mathrm{KMC}$ as relaxed, calm, happy, natural, instinctive, and safe [25-30]. Parents reported that the bonding associated with KMC felt connected, familiarizing, comforting, and logical. Mothers preferred KMC to traditional incubators because they felt closer to their babies, and it put them at ease [31].

\section{Social support for caregivers}

Social support referred to the perception and reality that one has assistance from other people to perform KMC. While practicing KMC, mothers and fathers did not feel supported by their families or communities $[15,25]$. Mothers experienced a lack of support from healthcare workers. Some hospital staff were resistant to family participation in caring for the baby while in the hospital [32]. Healthcare workers were occasionally considered to be loud and uncaring by parents [33, 34]. Additionally, KMC was impaired when parents perceived that HCWs did not respect family privacy [35]. Fathers reported lack of support from society and frequently voiced discomfort about performing KMC because of societal norms, as many fathers felt that childcare should be the role of the mother [15, 36]. Older generations, mothers-in-law, and grandmothers in particular, did not find $\mathrm{KMC}$ to be an appropriate method to care for newborns [37].

In contrast, KMC uptake was promoted by societal acceptance of paternal participation in childcare, by family and community acceptance of $\mathrm{KMC}$, and by the presence of engaged HCWs [38, 39]. In societies where gender roles were more equal, there were fewer barriers to fathers performing KMC $[39,40]$. Paternal involvement played a large role in KMC uptake-either by division of labor or by helping the mother feel comfortable [41]. Mothers were grateful to have someone help them during $\mathrm{KMC}$, such as grandmothers and sisters, who could take care of housework and help with the newborn. Within the maternity ward, peer support from other 


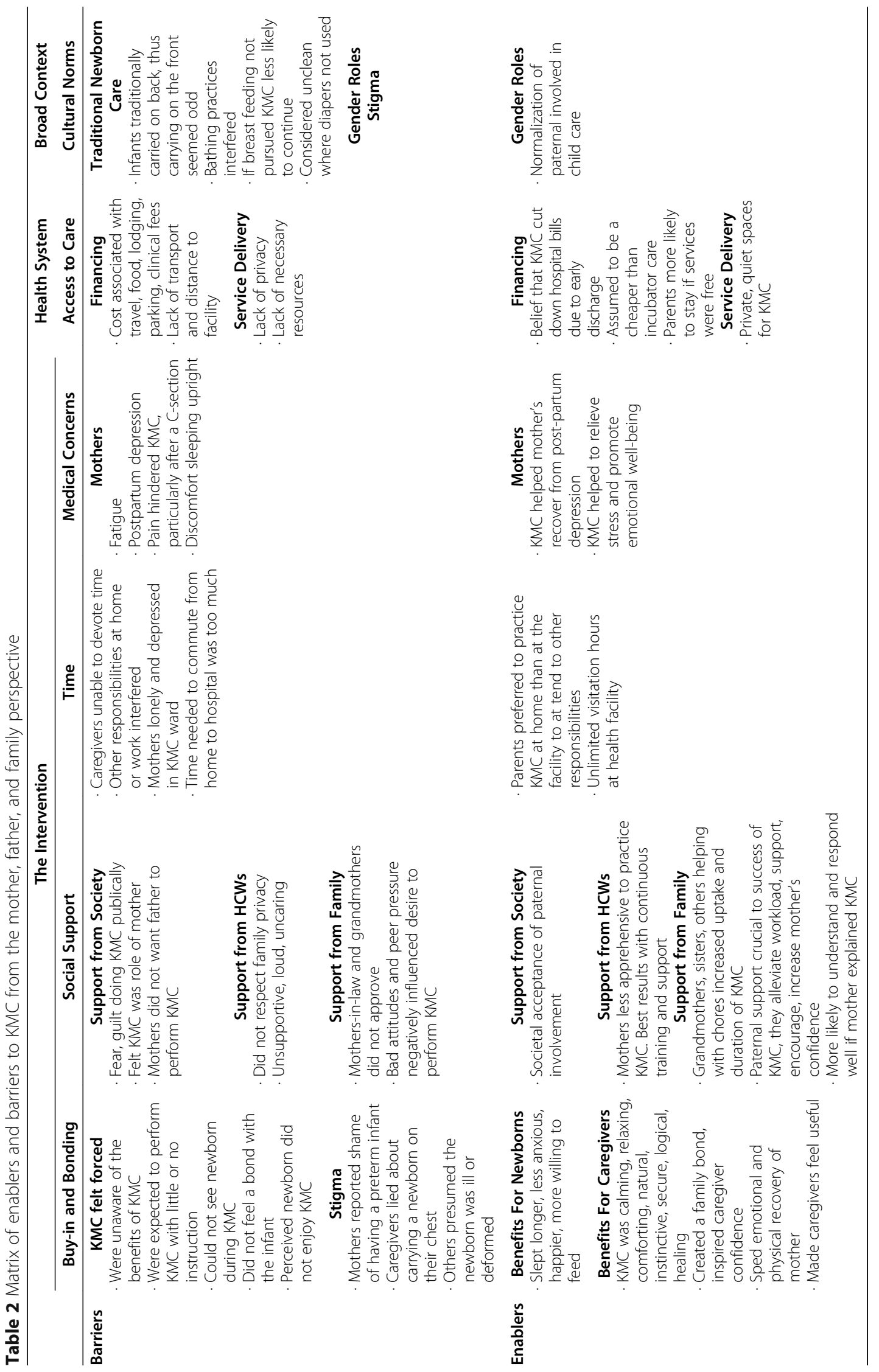


mothers who shared their $\mathrm{KMC}$ experiences also promoted acceptance [36, 42]. Additionally, the presence of well-trained nurses reduced maternal apprehension about practicing KMC and handling their newborn, facilitating the implementation of $\mathrm{KMC}$ [43].

\section{Caregiver time for KMC adoption}

KMC guidelines recommend continuous SSC for as long as possible until the newborn reaches a certain weight (usually $2000 \mathrm{~g}$ ), certain age (usually 2 weeks after birth), or no longer tolerates it [4]. The lengthy time needed to provide KMC was a barrier for caregivers. KMC was difficult to perform at long intervals if the mother was depressed, lonely, or recently had a C-section [44]. Many mothers found KMC performance at home to be a burden due to other responsibilities at home or work. For example, one mother said "Obviously I've got a husband and another child at home, and obviously have to cook...you have to clean and do a lot of other things, besides looking after yourself and the baby" (Mother) [42]. Another mother noted: 'Although I am very satisfied with the KMC method, it made me feel divided, as I was unable to be close to my other child. This must be even more complicated for those whose infant needs a longer period of hospital stay." (Mother) [27]. Another difficulty was commuting between home and KMC wards $[17,18,27,36,39,42,45,46]$. Thus, the ability to practice $\mathrm{KMC}$ at home, rather than in a facility, promoted the uptake of $\mathrm{KMC}$ by allowing caregivers to attend to other chores [47]. Parents (as well as staff) noted that unlimited visitation hours enabled adoption of KMC. Furthermore, facility staff felt as though parents were less interfering when they were allowed unhindered access to their babies [48].

\section{Caregiver medical concerns}

Medical concerns, including the clinical condition of the mother or newborn, may also be a barrier that prevents KMC uptake. For examples, mothers in Ghana found SSC problematic because they fear that by touching the umbilical cord of the newborn it would "divide into two," and cause pain, bleeding, or sickness [49]. Clinical consequences of $\mathrm{KMC}$ for mothers included fatigue, depression, and postpartum pain. Some mothers experienced discomfort sleeping upright with a newborn in KMC position $[13,39]$. Postpartum pain was considered a hindrance to SSC, especially after a C-section [29, 39, 49, 50]. However, women practicing $\mathrm{KMC}$ thought it helped them to recover from postpartum depression [51]. Mothers seemed to be satisfied with the method and felt that it helped relieve stress [31, 52].

\section{Health system barriers and enablers for caregiver adoption of KMC}

Adoption of KMC by caregivers generally begins in the context of the health system, and caregivers may interact with any of the core components of a health system. We found that financing and service delivery were aspects of the health system that influenced caregiver adoption of KMC.

\section{Financing}

First, in the case that the newborn remained in the hospital after the mother was discharged, lack of money for transportation and the distance to the hospital were often reported as the biggest challenges to KMC implementation; these were also barriers to returning to the health facility for follow up after both mother and infant were discharged but continuing KMC [53-56]. In an evaluation of a Kangaroo Care inpatient ward of a tertiary hospital in Malawi, 10\% mothers whose children died reported the distance to the health facility or lack of transport money as the reason they did not go the hospital when something was wrong with their newborn; similarly, nearly $40 \%$ of mothers reported lack of transport money as the reason they did not go the hospital for their follow up clinic appointments [56]. In Kuala Lumpur, Malaysia, poor public transportation and the difficulty of returning to the hospital after restarting work were the most frequently mentioned challenges to performing skin-to-skin contact on a daily basis while the newborn was still hospitalized [55]. On the other hand, free medical service enabled parents to stay at the clinic longer as needed. Also, parents in Harare, Zimbabwe believed that KMC decreased the cost of hospital bills and assumed that it was a cheaper option than conventional incubator care or a prolonged hospital stay [15].

\section{Service delivery}

For caregivers, lack of privacy and KMC resources at facilities presented obstacles to KMC adoption (Appendix). Structurally, there was a lack of private space for mothers to perform KMC and a lack of space for mothers to remain in the hospital with the newborn $[57,58]$. Mothers felt uncomfortable and exposed as staff continued to come in and out during KMC [25]. For example, "There were always people around. It is harder (to be skin to skin) when there are people other people coming in and out. Private rooms will help." (Mother) [50]. Another mother reported: "From seven in the morning until five in the afternoon people came in all the time. People came in to clean the room, clean the bathroom, to check on me and someone else to check on the baby. Every fifteen minutes someone different would come in. I could never relax. It was exhausting. It was stressful. I couldn't relax." (Mother) [50]. Lack of resources at facilities (e.g. chairs, beds, linens, 
curtains, KMC wraps, etc.) was also a barrier to adoption. At one facility materials donated for KMC were put into VIP units rather than the KMC ward [54].

However, the provision of private spaces, a quiet atmosphere, and dedicated resources promoted the acceptance and uptake of KMC [34]. Privacy screens or private rooms allowed the family separation from hospital staff and other patients and offered a quieter atmosphere for the mothers to conduct KMC [59].

\section{Social and cultural barriers and enablers for caregiver adoption of KMC}

We hypothesized that the broad social context (e.g. demographic, economic, and cultural factors) influence caregiver adoption of KMC. For example, surveys from 15 lowincome countries noted that health care professionals often found that KMC was thought of as substandard or as "the poor man's alternative" [36]. Also, caregiver adherence to traditional newborn practices was reported as a barrier to KMC [55]. Traditional early bathing behavior was seen as having numerous benefits and was identified as an ingrained behavior by studies conducted in Ghana and Bangladesh $[17,60]$. One traditional birth attendant noted: "The child needs to be bathed immediately in order to shape the head because whenever a child is delivered the head is very flat so you need to sharpen it to make it round" [60]. A mother who had recently delivered noted that "Babies are normally bathed shortly after birth because it will help them feel clean and healthy" [60]. Other traditional practices, such as sleeping by a lamp and smearing the baby with oil, make uptake of KMC more difficult. In reports from Ghana and Malawi, where carrying the baby on the back was common, it seemed strange to place the baby on the front, as instructed by KMC. One woman explained: "The back is stronger than the front and better for carrying" [49]. In some contexts, it was considered unclean to have the mother carry the baby on her chest without a diaper [36]. Stigma surrounding having a preterm infant can be severe and can act as a barrier to continued practice of KMC.

Different approaches to gender roles, the role of parents in childcare, the role of men in the household, and the roles of other family members also influenced KMC uptake $[15,32,36]$. For example, some fathers reported feeling uncomfortable practicing $\mathrm{KMC}$ in public, learning how to perform KMC while other people were present, or being scrutinized by nurses [42, 61]. Additionally, in some cases, mothers and traditional birth attendants reported feeling uncomfortable with the father performing their duties [25].

\section{Discussion}

Kangaroo mother care is a complex intervention, as defined in our conceptual framework, because user (i.e. caregiver) engagement is high and caregiver (i.e. the primary 'adoption system') behavior dominates our definition of 'successful implementation' of the intervention [8]. Thus, scale-up of this intervention around the world relies heavily on enabling caregivers to successfully adopt KMC. We found that buy-in and bonding, social support, time, and medical concerns were major themes defining the interaction between families and the KMC intervention. Furthermore, we identified financing and service delivery as barriers (and potential enablers) of KMC adoption within the context of the health system. Additionally we identified social and cultural norms that played an important role in the adoption of KMC.

Efforts to implement and scale up KMC must work to ensure a positive experience for caregivers. For example, the benefits of KMC to the newborn and caregivers must be clearly explained to everyone involved. One approach is to ensure healthcare workers present information about KMC in a standardized manner to caregivers and extended families, with attention paid to their concerns. Additionally, testimonials about the effectiveness of KMC could be given by caregivers who have successfully cared for a preterm or low birthweight baby in the past; such an approach is recommended in the Maternal and Child Health Integrated Program (MCHIP) KMC Guide [62]. Demonstrations and supervised practice can enhance caregiver confidence. Approaches to enhance newborn-caregiver bonding are needed. For example, programs might create a song about KMC to be sung to the baby, or healthcare workers could demonstrate the change in infant temperature after a period of skin to skin contact [63]. Despite efforts and ideas from programs and practitioners about how to create a positive KMC experience for caregivers, there is limited evidence about which approaches are effective.

We found that social support can enhance the uptake and duration of KMC. To enhance social support and promote positive attitudes about KMC, the Maternal and Child Health Integrated Program (MCHIP) KMC Guide recommends that programs undertake sensitization to $\mathrm{KMC}$ at the national, health facility, and community levels. At the community-level, recommended activities include celebrations for the 'graduation' of a baby from KMC or discussions about KMC through radio or other public forums [62]. Similarly, a program in Malawi asked respected grandparents to promote KMC and newborn care behavior. In the Agogo (the Chitumbuka word for grandparent) Program, the Ekwenedni Church of Central Africa Presbyterian (CCAP) Mission Hospital trained nearly 4000 grandparents. Subsequently, grandparents provided individual and group counseling in their respective villages, using drama, song, and poems 
to share key messages. An evaluation of the program concluded that grandparents were successful in promoting behavior change surrounding maternal and newborn care [64]. Encouraging family members to provide support by assisting mothers with other household responsibilities or coaching mothers how to ask for this support may also increase duration of KMC after hospital discharge. Currently, there is limited evidence about the effectiveness of such sensitization efforts.

Interactions between healthcare workers and families may either encourage or discourage caregiver adoption of KMC. This is consistent with research which demonstrated that interpersonal healthcare worker behavior is a significant contributor to patient satisfaction with maternal health services, which subsequently influences service utilization [65-67]. Healthcare workers and facilities can be supportive in their words and actions, by providing privacy for the family as they learn KMC and by ensuring unlimited visitation hours so that $\mathrm{KMC}$ can happen without time or schedule constraints. Health system concerns regarding financing travel, food, lodging, etc. may be partially alleviated by ensuring early discharge of mother and infant from the hospital (which should always be included as a component of $\mathrm{KMC}$ ). Additionally, KMC programs may consider ways to reduce hospital charges or provide transportation vouchers for families of infants with longer-thanaverage stays. For example, some programs in Colombia maintain social funds to provide financial support to families who must travel to a health facility in the period of close follow up after the newborn is discharged from the hospital (personal communication with Nathalie Charpak, Director Fundación Canguro). Automated cash transfers using cell phone technology, might be a method to reduce financial barriers. Transportation and time costs may also be addressed by offering home visits by community health workers for infant follow up. Further studies are needed to generate evidence regarding the feasibility and effectiveness of such an approach.

It is important to acknowledge that mothers, fathers, and families are adopting KMC within a broader social context. Several studies specifically noted that there may be stigma associated with having a preterm infant or around male involvement in child care, which present barriers to KMC uptake. Divisions of labor and space by gender have been found to be barriers to male participation in newborn care, in general. However, as Dumbaugh et al note, inclusion of men in newborn care must be done in a way that is empowering for women [68]. To address the reluctance of fathers to engage in childcare, fathers successfully engaging in SSC might become peer-mentors or demonstrators for other families. The intervention name "Kangaroo Mother Care" might also be changed so that it does not directly imply the behavior is performed only by the mother. Additional research about how to encourage paternal involvement and reduce stigma surrounding these childcare strategies must be a part of KMC scale up in any context.

\section{Strengths \& limitations}

The primary strength of this research is that it draws on the rich body of qualitative research that can help policy-makers and public health professionals to understand the complex context in which this intervention is implemented. However, our conclusions are limited by the existing body of evidence. There has been less research conducted in Southeast Asia and sub-Saharan Africa where KMC has the potential make the greatest impact. Furthermore, nearly half of the studies were conducted in urban settings with low neonatal mortality ( $<15$ per 1000 live births). Additional research is needed in the places where KMC has the potential for the highest impact and should be geared towards understanding the needs of caregivers of preterm and low birthweight infants. Future research should also investigate ways to generate demand for KMC services.

\section{Conclusion}

We found that lack of buy-in, poor social support, lack of time at the hospital or at home, and medical concerns about the mother or infant were barriers to caregiver adoption of KMC. Furthermore, we identified barriers and enablers of KMC adoption by families within the context of the health system and the broader social context. Future efforts to integrate KMC into local, regional, or national health systems must make efforts to identify and reduce barriers and promote enablers for successful caregiver adoption of KMC. Ultimately, KMC programs must ensure that the $\mathrm{KMC}$ experience is a valuable and positive experience from the caregiver perspective.

\section{Appendix}

Abbreviations
KMC: Kangaroo mother care; SSC: Skin-to-skin contact

\section{Acknowledgments}

We thank Ellen Boundy, Roya Dastjerdi, Sandhya Kajeepeta, Stacie Constantian, and Amy Labar for reviewing and abstracting the data, and Rodrigo Kuromoto and Eduardo Toledo for reviewing non-English articles. We acknowledge Kate Lobner for developing and running the search strategy.

\section{Funding}

This publication was supported by Save the Children's Saving Newborn Lives program. 


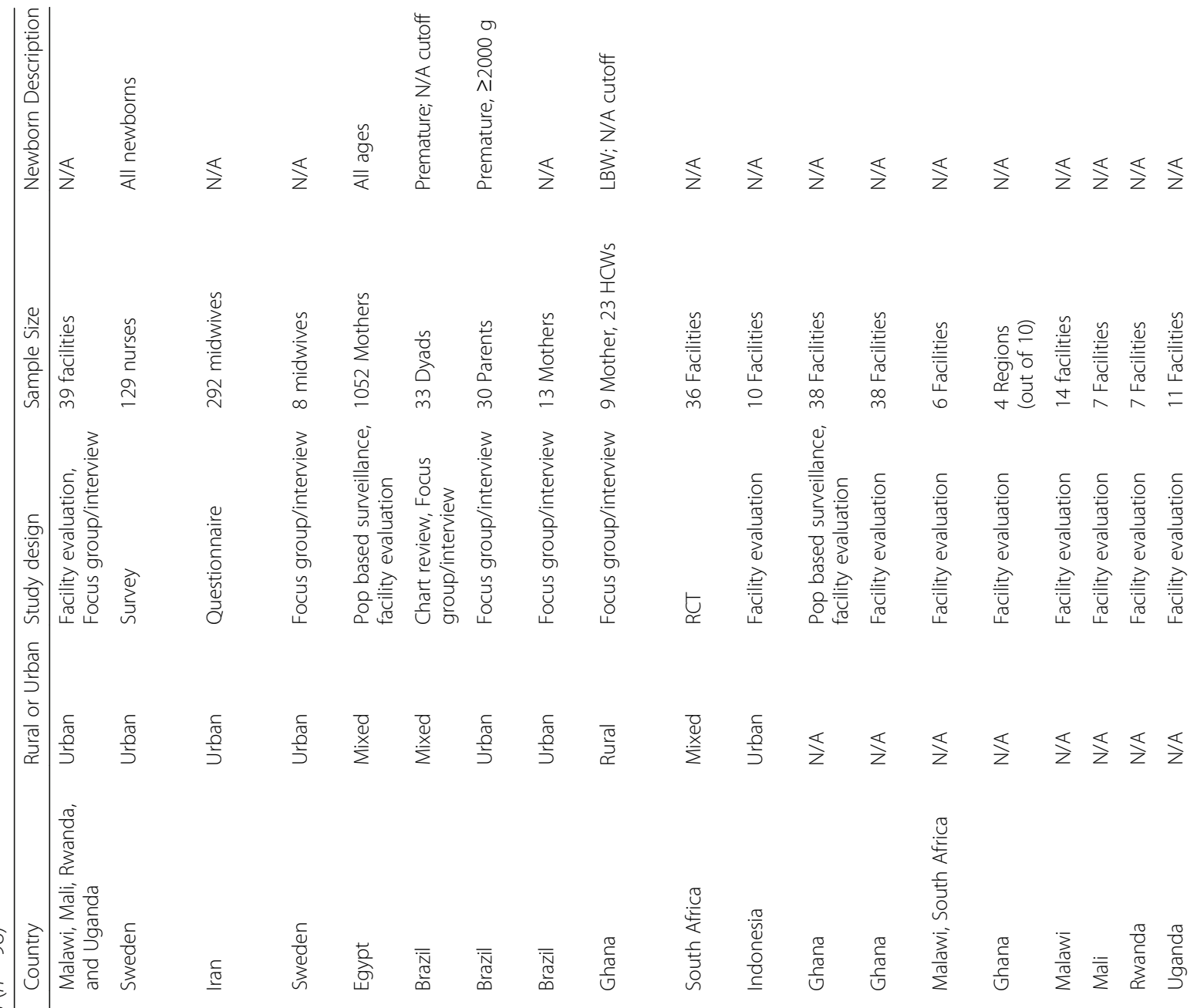

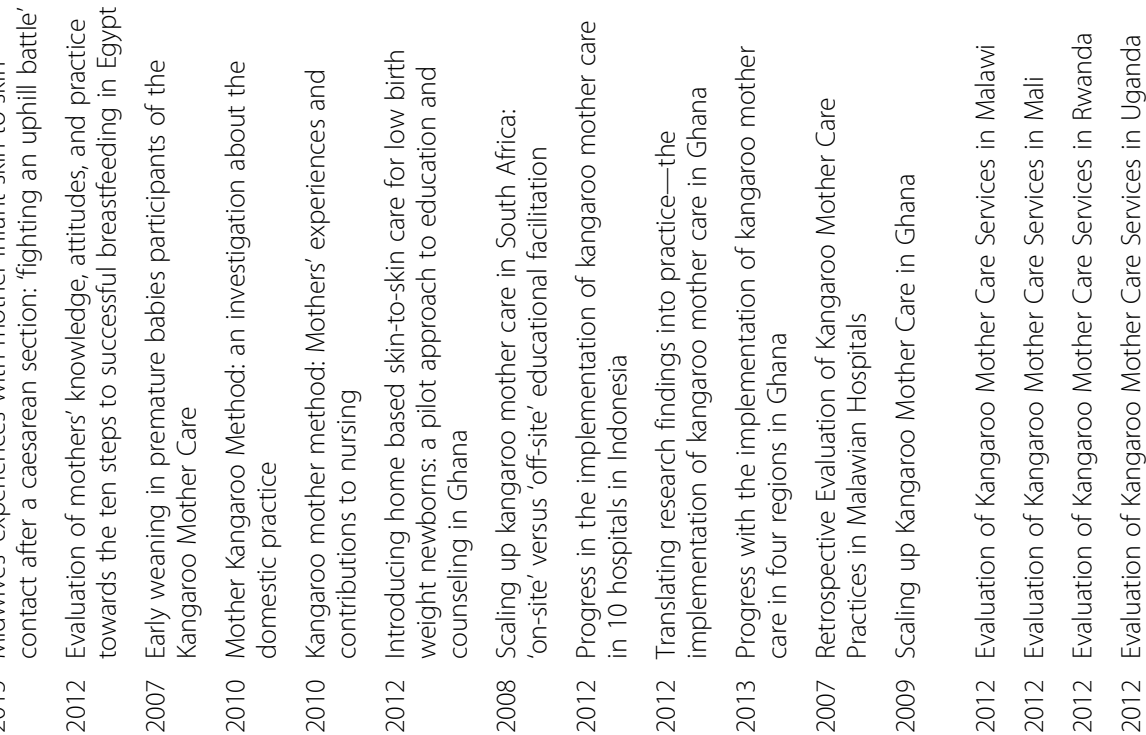




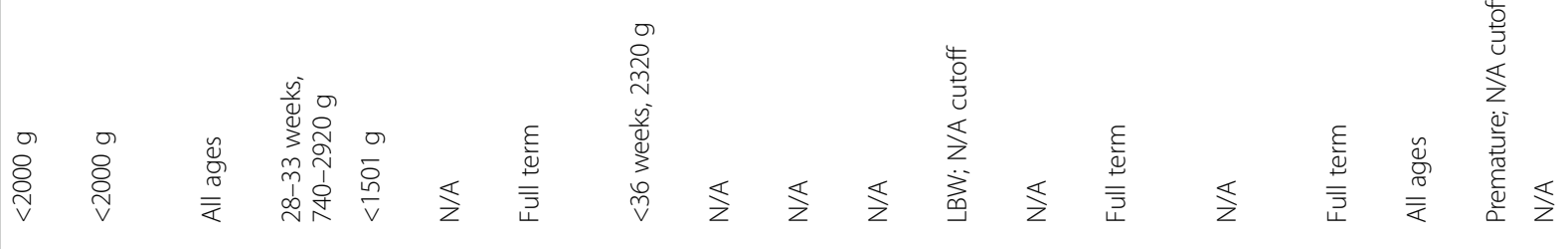

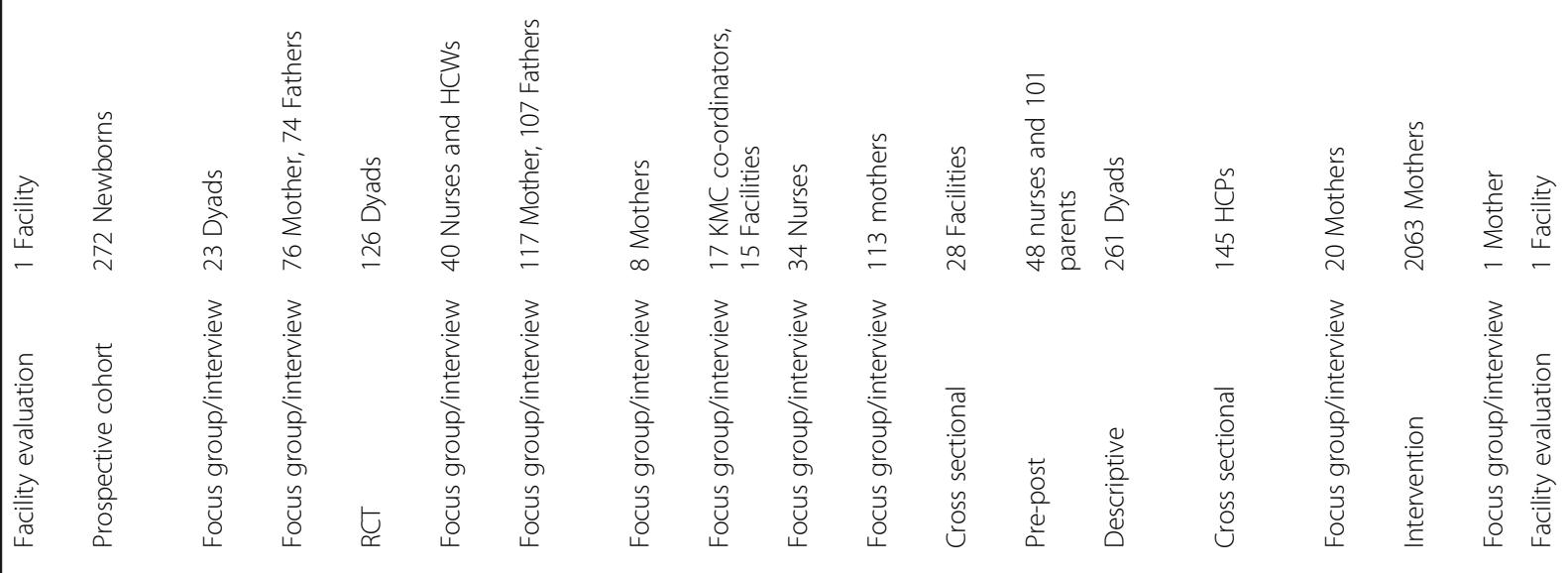

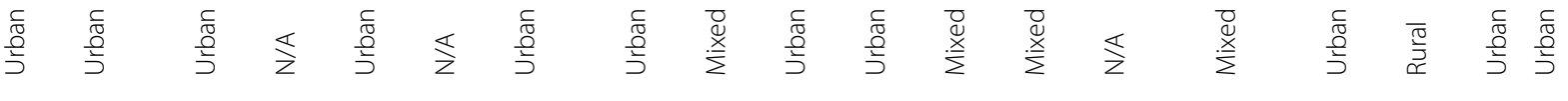

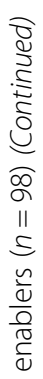

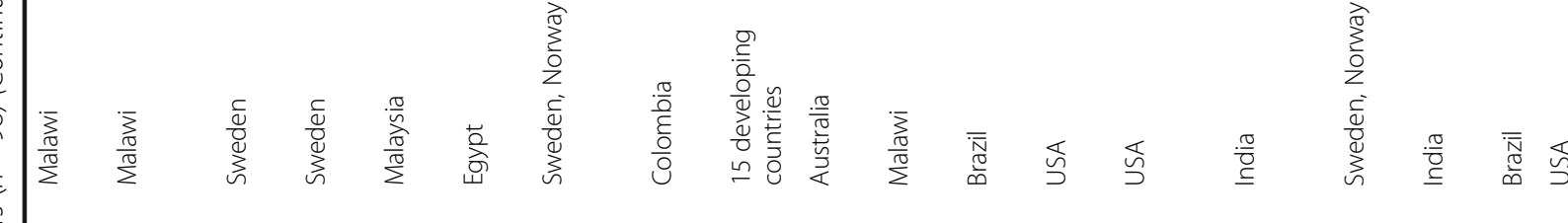




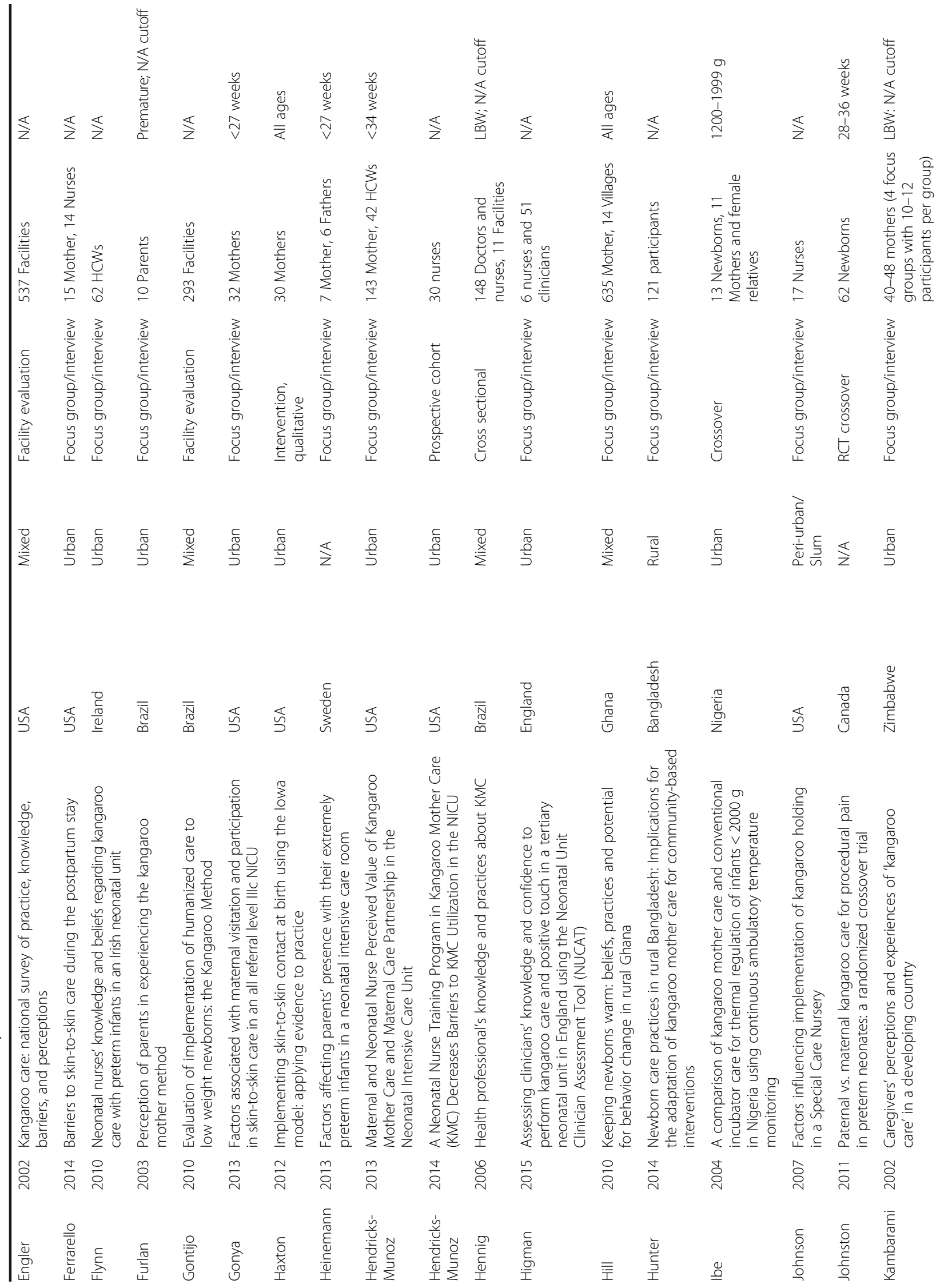




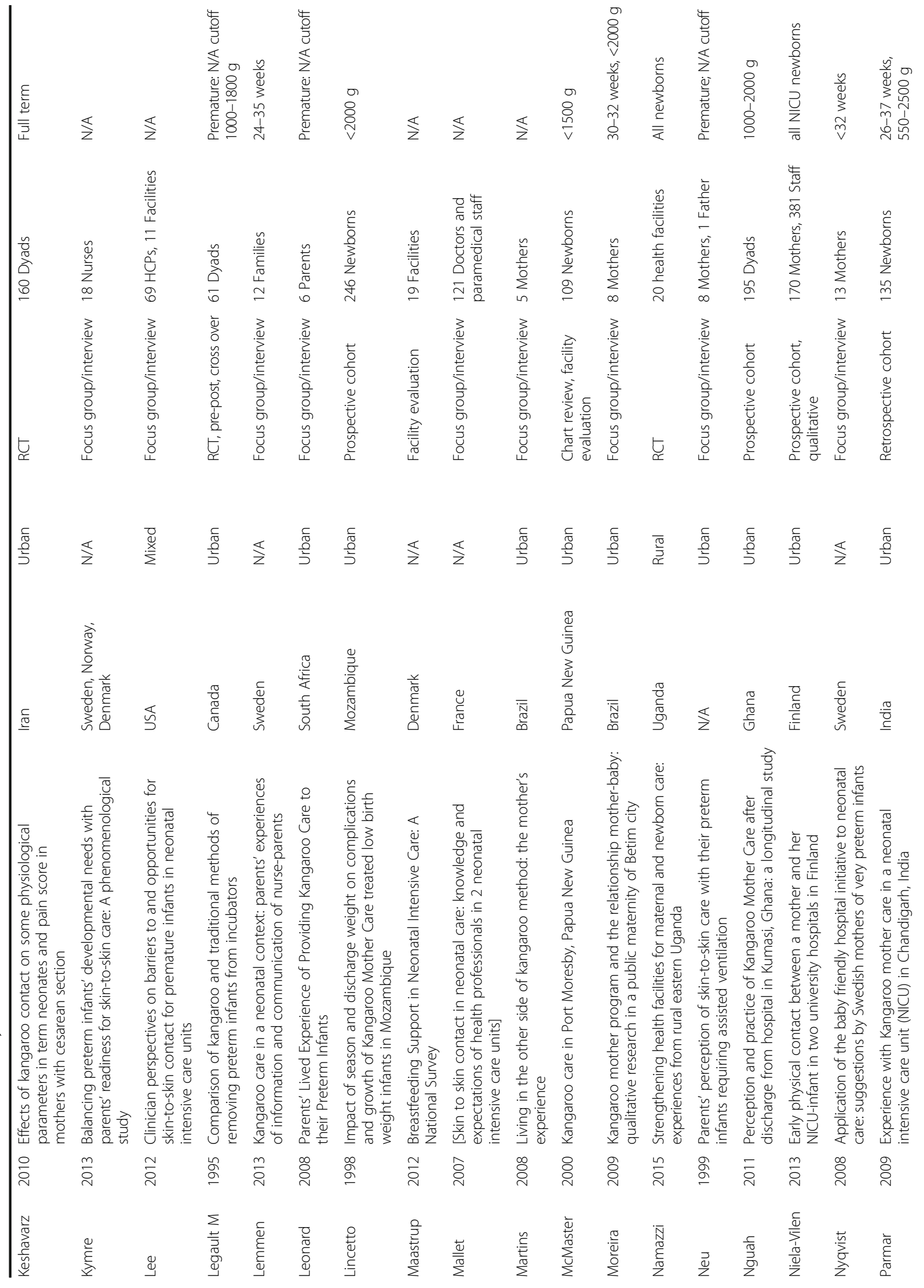




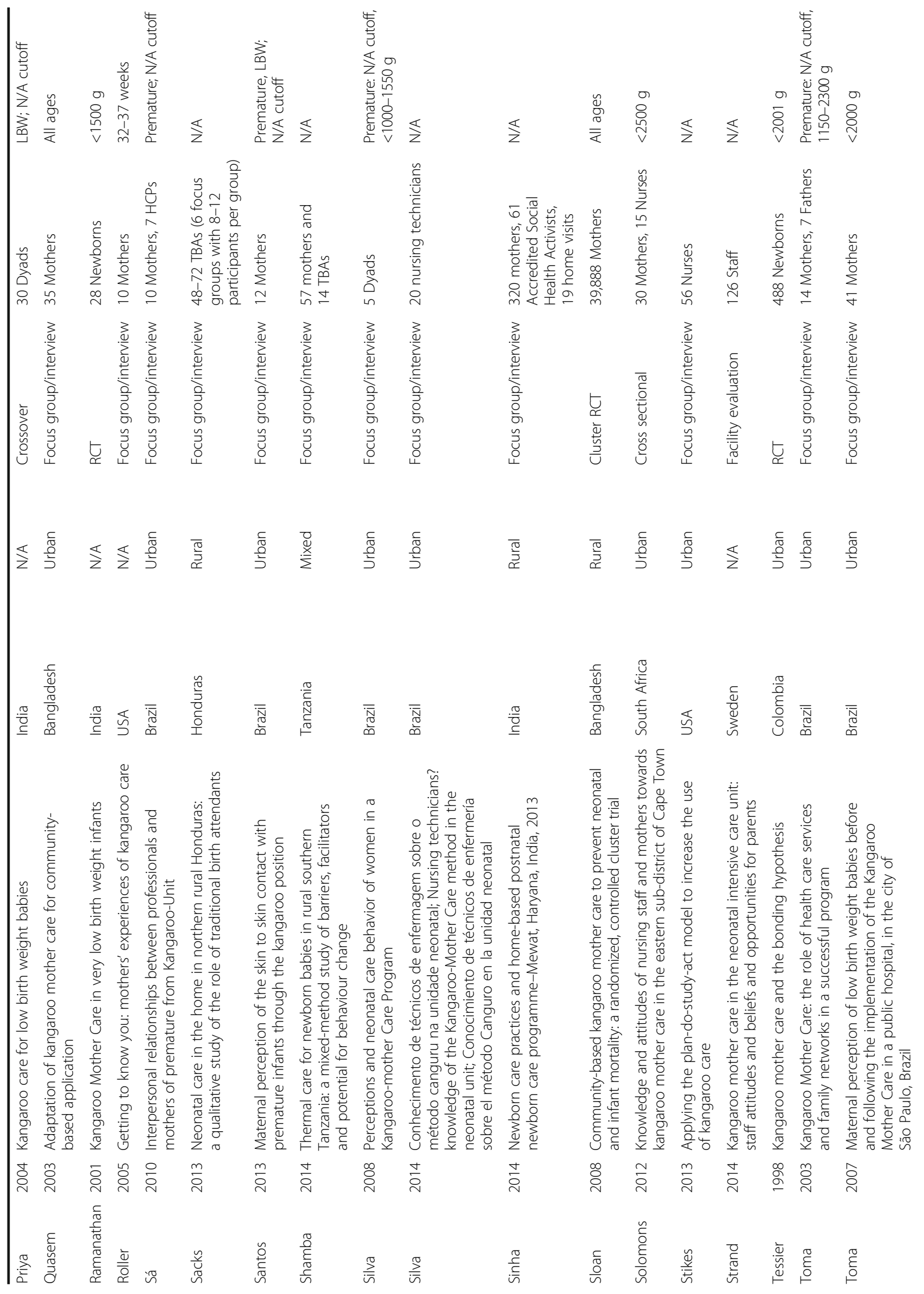




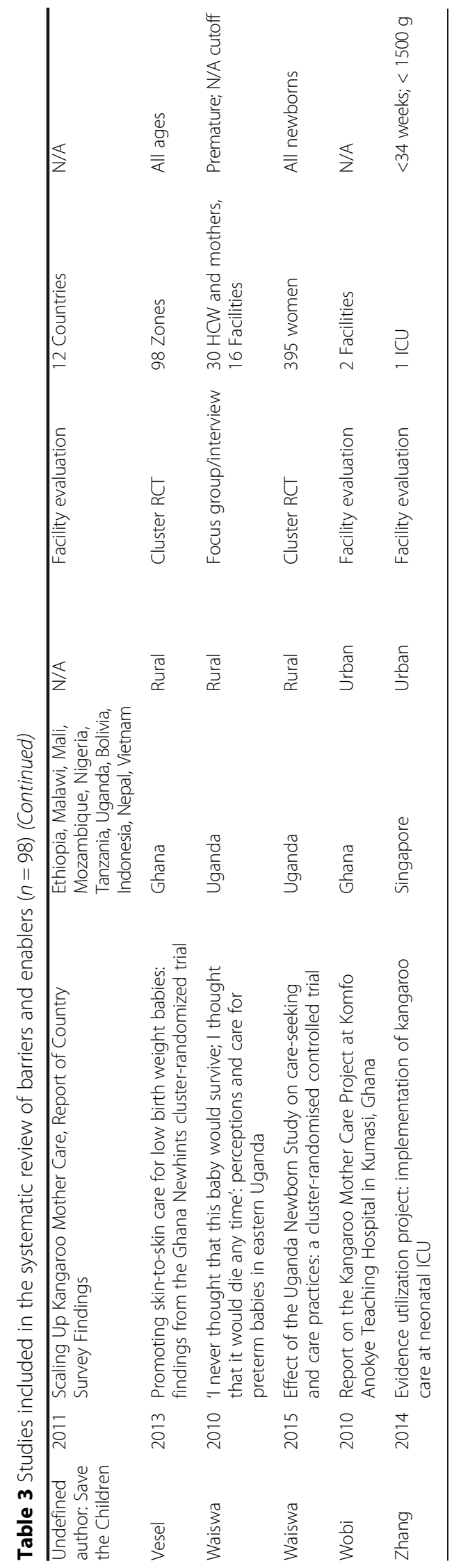




\section{Availability of data and materials}

Not applicable.

\section{Authors' contributions}

GC conceptualized and designed the review. IB, SC reviewed and abstracted data. ES, GC drafted the manuscript. All authors edited and reviewed the manuscript. All authors read and approved the final manuscript.

\section{Competing interests}

The authors declare that they have no competing interests.

\section{Consent for publication}

Not applicable.

\section{Ethics approval and consent to participate}

Not applicable.

\section{Author details}

'Department of Global Health and Population, Harvard T. H. Chan School of Public Health, 665 Huntington Ave., Building 1, Boston, MA 02115, USA. ${ }^{2}$ Division of Medicine Critical Care, Boston Children's Hospital, Boston, MA USA. ${ }^{3}$ Saving Newborn Lives, Save the Children, Washington, D.C., USA.

\section{Received: 16 August 2016 Accepted: 30 December 2016}

\section{Published online: 25 January 2017}

\section{References}

1. Wang H, Liddell CA, Coates MM, Mooney MD, Levitz CE, Schumacher AE, Apfel H, lannarone M, Phillips B, Lofgren KT, et al. Global, regional, and national levels of neonatal, infant, and under-5 mortality during 19902013: a systematic analysis for the Global Burden of Disease Study 2013. Lancet. 2014;384(9947):957-79.

2. Bhutta ZA, Black RE. Global maternal, newborn, and child health—so near and yet so far. N Engl J Med. 2013;369(23):2226-35.

3. World Health Organization DoRHaR. Kangaroo mother care: a practica guide. In: WHO Library. Geneva: World Health Organization; 2003.

4. Chan GJ, Valsangkar B, Kajeepeta S, Boundy EO, Wall S. What is kangaroo mother care? A systematic review of the literature. J Glob Health. 2016. In press.

5. Boundy EO, Dastjerdi R, Spiegelman D, Fawzi WW, Missmer SA, Lieberman E, Kajeepeta S, Wall S, Chan GJ. Kangaroo Mother Care and Neonatal Outcomes: A Meta-analysis. Pediatrics. 2016;137(1):1-16.

6. WHO. WHO recommendations on interventions to improve preterm birth outcomes. In. Geneva: World Health Organization. (2015).

7. Chan GJ, Labar AS, Wall S, Atun R. Kangaroo mother care: a systematic review of barriers and enablers. Bull World Health Organ. 2016:94(2):130-41

8. Atun R, de Jongh T, Secci F, Ohiri K, Adeyi O. Integration of targeted health interventions into health systems: a conceptual framework for analysis. Health Policy Plan. 2010;25(2):104-11.

9. Kuper A, Lingard L, Levinson W. Critically appraising qualitative research. BMJ. 2008;337:a1035

10. Kastner M, Antony J, Soobiah C, Straus SE, Tricco AC. Conceptual recommendations for selecting the most appropriate knowledge synthesis method to answer research questions related to complex evidence. J Clin Epidemiol. 2016;73:43-9.

11. Lemmen DF, Fristedt $P$, Lundqvist A. Kangaroo care in a neonatal context: Parents' experiences of information and communication of Nurse-Parents. Open Nurs J. 2013;7:41-8.

12. Martins AJVS, Santos IMM. Living in the other side of kangaroo method: the mother's experience. Rev Eletr Enf 10.3. 2008.

13. Quasem I, Sloan NL, Chowdhury A, Ahmed S, Winikoff B, Chowdhury AM. Adaptation of kangaroo mother care for community-based application. J Perinatol. 2003;23(8):646-51.

14. Brimdyr K, Widstrom AM, Cadwell K, Svensson K, Turner-Maffei C. A Realistic Evaluation of Two Training Programs on Implementing Skin-to-Skin as a Standard of Care. J Perinat Educ. 2012;21(3):149-57.

15. Kambarami RA, Mutambirwa J, Maramba PP. Caregivers' perceptions and experiences of 'kangaroo care' in a developing country. Trop Dr. 2002;32(3):131-3.

16. Duarte ED, Sena RR. Kangaroo mother care: experience report. Revista Mineira de Enfermagem. 2001;5(1):86-92.
17. Waiswa P, Nyanzi S, Namusoko-Kalungi S, Peterson S, Tomson G, Pariyo GW. 'I never thought that this baby would survive; I thought that it would die any time': perceptions and care for preterm babies in eastern Uganda. Trop Med Int Health. 2010;15(10):1140-7.

18. Dalbye R, Calais E, Berg M. Mothers' experiences of skin-to-skin care of healthy fullterm newborns-A phenomenology study. Sex Reprod Healthc. 2011;2(3):107-11.

19. Neu M. Parents' perception of skin-to-skin care with their preterm infants requiring assisted ventilation. J Obstet Gynecol Neonatal Nurs. 1999;28(2):157-64.

20. Crenshaw JT, Cadwell K, Brimdyr K, Widstrom AM, Svensson K, Champion JD, Gilder RE, Winslow EH. Use of a video-ethnographic intervention (PRECESS Immersion Method) to improve skin-to-skin care and breastfeeding rates. Breastfeed Med. 2012;7(2):69-78.

21. Keshavarz M, Haghighi NB. Effects of kangaroo contact on some physiological parameters in term neonates and pain score in mothers with cesarean section. Koomesh. 2010;11(2):91-9.

22. Johnston CC, Campbell-Yeo M, Filion F. Paternal vs maternal kangaroo care for procedural pain in preterm neonates: a randomized crossover trial. Arch Pediatr Adolesc Med. 2011;165(9):792-6.

23. Ludington-Hoe SM, Johnson MW, Morgan K, Lewis T, Gutman J, Wilson PD, Scher MS. Neurophysiologic assessment of neonatal sleep organization: Preliminary results of a randomized, controlled trial of skin contact with preterm infants. Pediatrics. 2006;117(5):E909-23.

24. Tessier R, Cristo MB, Velez S, Giron M, Nadeau L, Figueroa de Calume Z, Ruiz-Palaez JG, Charpak N. Kangaroo Mother Care: A method for protecting high-risk low-birth-weight and premature infants against developmental delay. Infant Behav Dev. 2003;26(3):384-97.

25. Sá FE, Sá RC, Pinheiro LMF, Callou FEO. Interpersonal relationships between professionals and mothers of premature from Kangaroo-Unit. Revista Brasileira em Promoção da Saúde. 2010;23(2):144-9.

26. Roller CG. Getting to know you: mothers' experiences of kangaroo care. J Obstet Gynecol Neonatal Nurs. 2005;34(2):210-7.

27. Blomqvist YT, Nyqvist KH. Swedish mothers' experience of continuous Kangaroo Mother Care. J Clin Nurs. 2011;20(9-10):1472-80.

28. Heinemann AB, Hellstrom-Westas L, Hedberg Nyqvist K. Factors affecting parents' presence with their extremely preterm infants in a neonatal intensive care room. Acta Paediatr. 2013:102(7):695-702.

29. Darmstadt GL, Kumar V, Yadav R, Singh V, Singh P, Mohanty S, Baqui AH, Bharti N, Gupta S, Misra RP, et al. Introduction of community-based skin-to-skin care in rural Uttar Pradesh, India. J Perinatol. 2006;26(10):597-604.

30. Nyqvist $\mathrm{KH}$, Kylberg E. Application of the baby friendly hospital initiative to neonatal care: suggestions by Swedish mothers of very preterm infants. J Hum Lact. 2008;24(3):252-62.

31. Legault M, Goulet C. Comparison of kangaroo and traditional methods of removing preterm infants from incubators. J Obstet Gynecol Neonatal Nurs. 1995;24:501-6.

32. Colameo AJ, Rea MF. Kangaroo Mother Care in public hospitals in the State of Sao Paulo, Brazil: an analysis of the implementation process. Cad Saude Publica. 2006;22(3):597-607.

33. Blomqvist YTF, Frölund L, Rubertsson C, Nyqvist KH. Provision of Kangaroo Mother Care: Supportive factors and barriers perceived by parents. Scand J Caring Sci. 2013;27:345-53.

34. Heinemann AB, Hellstrom-Westas $L$, Nyqvist KH. Factors affecting parents' presence with their extremely preterm infants in a neonatal intensive care room. Acta Paediatr. 2013;102:695-702.

35. Neu M. Kangaroo care: is it for everyone? Neonatal Netw. 2004:23(5):47-54.

36. Charpak N, Ruiz-Pelaez JG. Resistance to implementing Kangaroo Mother Care in developing countries, and proposed solutions. Acta Paediatr. 2006;95(5):529-34.

37. Kambarami R. Kangaroo care and multiple births. Ann Trop Paediatr. 2002;22(1):107-8

38. Kambarami RA, Chidede O, Pereira N. Long-term outcome of preterm infants discharged home on kangaroo care in a developing country. Ann Trop Paediatr. 2003;23(1):55-9.

39. Blomqvist YT, Frölund L, Rubertsson C, Nyqvist KH. Provision of Kangaroo Mother Care: Supportive factors and barriers perceived by parents. Scand J Caring Sci. 2013;27:345-53.

40. Calais E, Dalbye R, Nyqvist K, Berg M. Skin-to-skin contact of fullterm infants: an explorative study of promoting and hindering factors in two Nordic childbirth settings. Acta Paediatr. 2010;99(7):1080-90.

41. Moreira JO, Romagnoli RC, Dias DAS, Moreira CB. Kangaroo mother program and the relationship mother-baby: qualitative research in a public maternity of Betim city. Psicol estud. 2009;14(3):475-83. 
42. Leonard A, Mayers P. Parents' Lived Experience of Providing Kangaroo Care to their Preterm Infants. Health SA Gesondheid. 2008;13(4):16-28.

43. Solomons $N$, Rosant $C$. Knowledge and attitudes of nursing staff and mothers towards kangaroo mother care in the eastern sub-district of Cape Town. S Afr J Clin Nutr. 2012;25(1):33-9.

44. Kymre IG, Bondas T. Balancing preterm infants' developmental needs with parents' readiness for skin-to-skin care: A phenomenological study. Int J Qual Stud Health Well-being. 2013;8(1):21370.

45. Toma TS, Venâncio SI, Andretto DA. Percepção das mães sobre o cuidado do bebê de baixo peso antes e após implantação do Método Mãe-Canguru em hospital público da cidade de São Paulo, Brasil. [Maternal perception of low birth weight babies before and following the implementation of the Kangaroo Mother Care in a public hospital, in the city of São Paulo, Brazil]. Rev Bras Saúde Matern Infant. 2007;7(3): 297-307.

46. Castiblanco López N, Muñoz de Rodríguez L. Vision of mothers in care of premature babies at home. Av enferm. 2011;29(1):120-9.

47. Ibe OE, Austin T, Sullivan K, Fabanwo O, Disu E, Costello AM. A comparison of kangaroo mother care and conventional incubator care for thermal regulation of infants $<2000 \mathrm{~g}$ in Nigeria using continuous ambulatory temperature monitoring. Ann Trop Paediatr. 2004;24(3):245-51.

48. De Vonderweid U, Forleo V, Petrina D, Sanesi C, Fertz C, Leonessa ML, Cuttini M. Neonatal developmental care in Italian Neonatal Intensive Care Units. Ital J Pediatr. 2003;29(3):199-205.

49. Bazzano A, Hill Z, Tawiah-Agyemang C, Manu A, ten Asbroek G, Kirkwood B. Introducing home based skin-to-skin care for low birth weight newborns: a pilot approach to education and counseling in Ghana. Glob Health Promot. 2012;19(3):42-9.

50. Ferrarello $\mathrm{DH}$, Hatfield L. Barriers to skin-to-skin care during the postpartum stay. MCN Am J Matern Child Nurs. 2014;39:56-61.

51. Silva MBO, Brito RCS. Perceptions and neonatal care behavior of women in a Kangaroo-mother Care Program. Interao Psicol. 2008;12(2):255-66.

52. Furlan CEFB, Scochi CGS, Furtado MCC. Percepção dos pais sobre a vivência no método mãe-canguru. Perception of parents in experiencing the kangaroo mother method. Rev Lat Am Enfermagem. 2003;11(4):444-52.

53. Bergh AM, et al. Retrospective evaluation of kangaroo mother care practices in Malawian hospitals. Healthy Newborn Network. 2007.

54. Bergh AM, Sylla M, Traore IMA, Diall Bengaly H, Kante M, Diaby Kaba N. Evaluation of kangaroo mother care services in Mali. 2012.

55. Boo NY, Jamli FM. Short duration of skin-to-skin contact: Effects on growth and breastfeeding. J Paediatr Child Health. 2007;43(12):831-6.

56. Blencowe $\mathrm{H}$, Kerac M, Molyneux E. Safety, effectiveness and barriers to follow-up using an 'early discharge' Kangaroo Care policy in a resource poor setting. J Trop Pediatr. 2009;55(4):244-8.

57. Bergh AM, Davy K, Otai CD, Nalongo AK, Sengendo NH, Aliganyira P. Evaluation of kangaroo mother care services in Uganda. 2012.

58. Bergh AM, Banda L, Lipato T, Ngwira G, Luhanga R, Ligowe R. Evaluation of kangaroo mother care services in Malawi. 2012.

59. Johnson AN. Factors influencing implementation of kangaroo holding in a Special Care Nursery. MCN Am J Matern Child Nurs. 2007;32(1):25-9.

60. Hill Z, Tawiah-Agyemang C, Manu A, Okyere E, Kirkwood BR. Keeping newborns warm: beliefs, practices and potential for behaviour change in rural Ghana. Trop Med Int Health. 2010;15(10):1118-24.

61. Eleutério FRR, Rolim KMC, Campos ACS, Frota MA, Oliveira MMC. The imaginary of mothers about experiencing the mother-kangaroo method. Cinc Cuid Saude. 2008;7(4):439-46

62. MCHIP. Kangaroo Mother Care: Implementation Guide. The Maternal and Child Health Integrated Program (MCHIP) of USAID. 2012.

63. Kumar V, Mohanty S, Kumar A, Misra RP, Santosham M, Awasthi S, Baqui AH, Singh P, Singh V, Ahuja RC, et al. Effect of community-based behaviour change management on neonatal mortality in Shivgarh, Uttar Pradesh, India: a cluster-randomised controlled trial. Lancet. 2008;372(9644):1151-62.

64. Zyl MV. The Ekwendeni Agogo Approach: Grandparents as agents of change for newborn survival. Healthy Newborn Network. 2010.

65. Kujawski S, Mbaruku G, Freedman LP, Ramsey K, Moyo W, Kruk ME. Association Between Disrespect and Abuse During Childbirth and Women's Confidence in Health Facilities in Tanzania. Matern Child Health J. 2015;19(10):2243-50.

66. Avortri GS, Beke A, Abekah-Nkrumah G. Predictors of satisfaction with child birth services in public hospitals in Ghana. Int J Health Care Qual Assur. 2011;24(3):223-37.
67. Larson E, Hermosilla S, Kimweri A, Mbaruku GM, Kruk ME. Determinants of perceived quality of obstetric care in rural Tanzania: a cross-sectional study. BMC Health Serv Res. 2014;14:483.

68. Dumbaugh M, Tawiah-Agyemang C, Manu A, ten Asbroek GH, Kirkwood B, Hill Z. Perceptions of, attitudes towards and barriers to male involvement in newborn care in rural Ghana, West Africa: a qualitative analysis. BMC Pregnancy Childbirth. 2014;14:269.

\section{Submit your next manuscript to BioMed Central and we will help you at every step:}

- We accept pre-submission inquiries

- Our selector tool helps you to find the most relevant journal

- We provide round the clock customer support

- Convenient online submission

- Thorough peer review

- Inclusion in PubMed and all major indexing services

- Maximum visibility for your research

Submit your manuscript at www.biomedcentral.com/submit
Biomed Central 